\title{
Investigation of vascular compression of the trachea: the complementary roles of barium swallow and echocardiography
}

\author{
Michael Burch, Seshadri Balaji, John E Deanfield, Ian D Sullivan
}

\begin{abstract}
To determine the preferred investigation of congenital vascular compression of the trachea, preoperative investigation in $\mathbf{4 0}$ patients undergoing surgery 1981-91 was analysed. The importance of preoperative identification of detailed arterial anatomy in determining surgical technique was also assessed in 122 consecutive patients undergoing surgery 1952-91. Of the 40 patients (median age 5 months) seen 1981-91, barium swallow before referral suggested the diagnosis of vascular compression in 26 of 27 (96\%). Angiography (1/3), bronchoscopy (0/6), and echocardiography (2/6) were generally unhelpful in diagnosing vascular compression before referral. Echocardiography was performed after referral in 18 consecutive patients since 1986 and the correct arterial anatomy was identified in $16(88 \%)$; hyperinflation resulted in inadequate images in the two others. Barium swallow is the investigation of choice to screen for suspected vascular compression of the trachea; echocardiography at the referral centre can define the arterial anatomy in nearly all patients. Further investigation can be reserved for selected cases.
\end{abstract}

(Arch Dis Child 1993;68:171-6)

\author{
Cardiothoracic Unit, \\ Hospital for \\ Great Ormond Street, \\ London WCIN 3JH \\ Michael Burch \\ Seshadri Balaji \\ John E Deanfield \\ Ian D Sullivan \\ Correspondence to \\ Dr Sullivan. \\ Accepted 14 May 1992
}
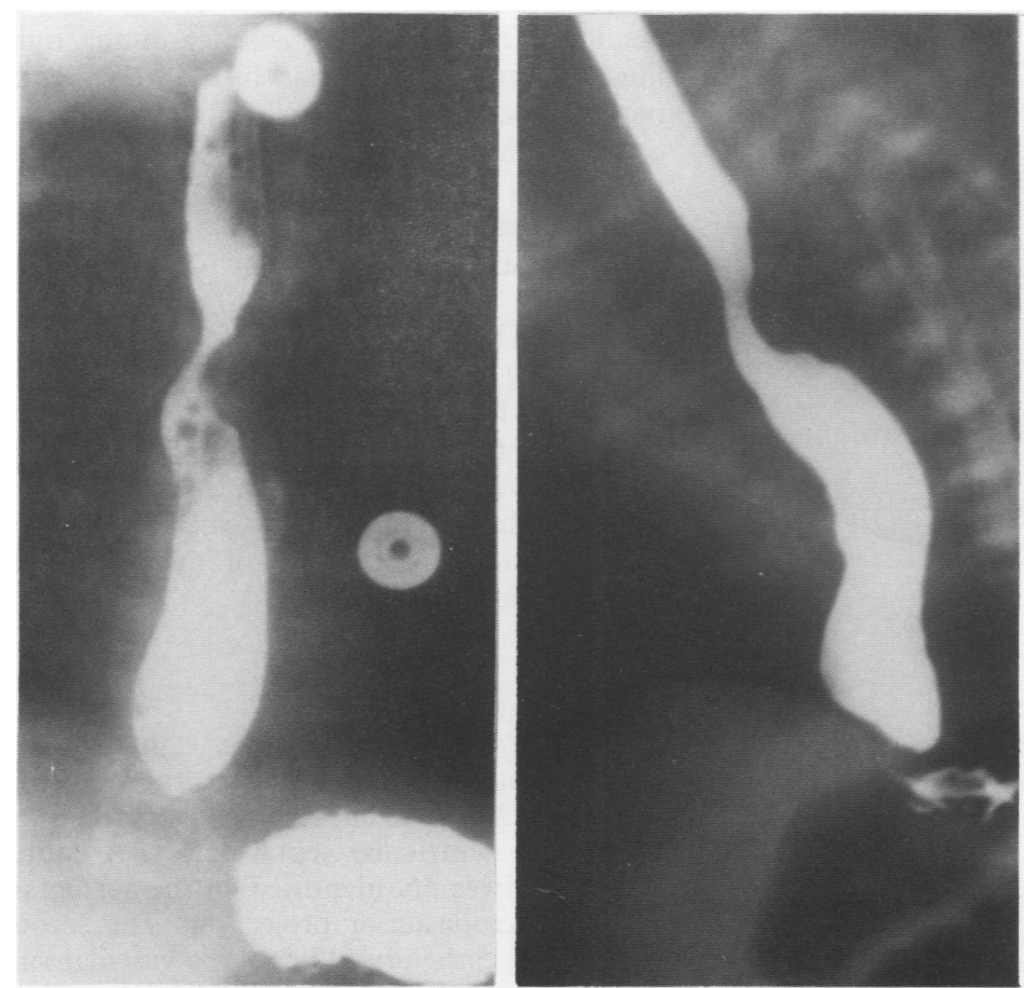

Figure 1 Barium swallow in a patient with double aortic arch. Bilateral indentation of the oesophagus is present on the anteroposterior view (left), with posterior indentation evident on the left anterior oblique view (right).
Vascular rings or slings that could cause tracheal or oesophageal compression in humans were demonstrated at postmortem examination almost 200 years ago. ${ }^{1}$ It was not until 1945 , however, that surgical division was described. ${ }^{2}$ Echocardiography can demonstrate vascular compression of the trachea, ${ }^{34}$ and case reports have emphasised the helpful recent addition of colour flow Doppler. ${ }^{5} 6$ However, neither the accuracy nor the limitations of the technique are known. Consequently, we have reviewed the patients who underwent surgery from 1981 and 1991 to examine the role of echocardiography, in conjunction with barium swallow and other imaging techniques, in an attempt to define the preferred method of preoperative assessment. In addition, we have combined these patients with those undergoing surgery between 1952 and 1980 to provide a 39 year single centre experience of consecutive patients with vascular compression of the trachea to determine the importance of accurate preoperative identification of arterial anatomy.

\section{Patients}

Preoperative investigation was analysed in 40 patients (median age 5 months, range 6 days8 years) who underwent surgery to relieve vascular compression of the trachea since January 1981. All but one had stridor, which characteristically had an expiratory component and was present from the first day of life in $26(65 \%)$. The one patient without stridor presented at 6 days with cyanosis related to associated tetralogy of Fallot. Nine (22\%) required intubation and ventilation preoperatively, although only three were ventilated at the time of transfer. An additional infant with a known diagnosis of double aortic arch was referred after operation in another hospital because of persistence of symptoms and is not included in this series. Detailed arterial anatomy and its influence on surgical technique was determined in all $12 \hat{z}$ patients undergoing surgery 1952-91.

\section{Results}

THE INVESTIGATION OF VASCULAR ANOMALIES 1981-91

\section{Barium studies}

Barium oesophograms (fig 1) were performed in 38 of the 40 patients $(95 \%)$. These were performed at the referring hospital in 27, although the barium studies were repeated in seven patients after referral. Vascular compression was suspected on all except one of the studies at the referring hospital and the correct anatomical diagnosis was made in 6/27 (22\%) (fig 2A). The 
vascular anomaly was correctly identified in a further $7 / 27(26 \%)$ on review of the films after referral. This included the patient in whom vascular compression had not been suspected before referral in whom a pulmonary artery sling was identified. The barium swallows performed at the Hospital for Sick Children (seven repeat, 11 initial studies), identified vascular compression in 16 of $18(89 \%)$ and the anatomy was correctly predicted in seven of 18 (39\%) patients (fig 2B). In 9/18 (50\%) a nonspecific diagnosis of 'vascular ring' was made. Two patients with pulmonary artery slings were ventilated at the time of transfer. In both cases an inadequate barium study was performed. One had the oesophogram performed via a nasogastric tube and the other had only a small volume of barium because of fear of aspiration. In neither case was vascular compression recognised. Overall, the anatomy was correctly diagnosed in specific conditions as follows: double aortic arch 16/25 (64\%), pulmonary artery sling $4 / 7$, left aortic arch with aberrant right subclavian artery $1 / 1$, and right aortic arch with aberrant left subclavian artery and left duct
A

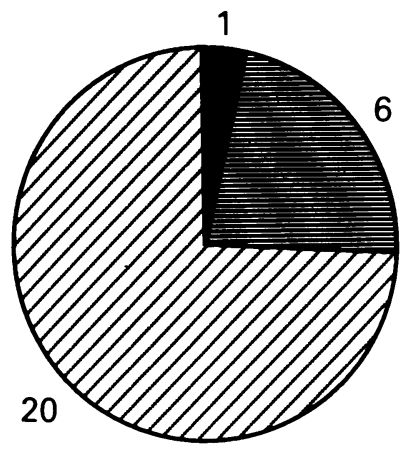

$n=27$

C

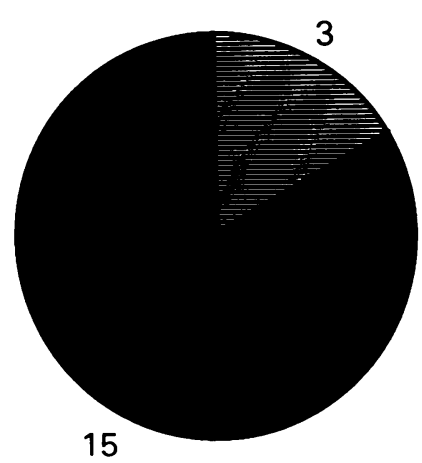

$\mathrm{n}=18$
B

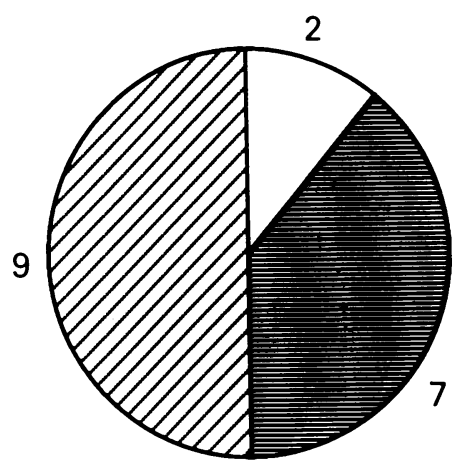

$n=18$

D

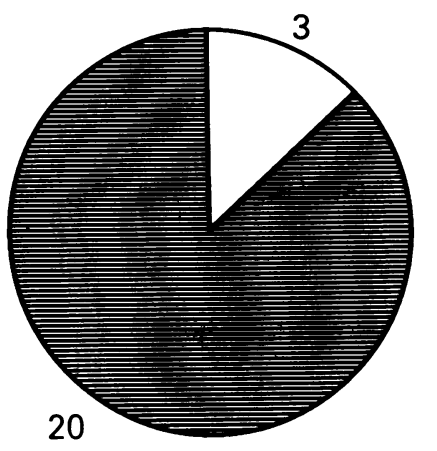

$n=23$ or ligament $0 / 5$. No false positive diagnosis of vascular ring was made on the basis of barium studies during this period.

\section{Echocardiography}

Echocardiography (fig 3) was first used in the assessment of these anomalies at the Hospital for Sick Children in 1982, but not routinely until 1986. Echocardiography was performed in $5 / 22$ patients seen $1981-5$ and all 18 patients seen 1986-91 (fig 2D). In one patient studied in 1983 adequate images to demonstrate the arch anatomy were not obtained and no attempt to define the lesion was made. Display of the arterial anatomy was incomplete in four others. The extreme superior and posterior origin of the left pulmonary artery was not clearly demonstrated in one patient with a pulmonary artery sling but the abnormal proximal course of the artery was identified. In a patient with a left aortic arch, there was no bifurcation of the first branch from the aorta and the aberrant origin of the right subclavian artery was diagnosed by inference. Colour flow Doppler, which has since facilitated identification of both pulmonary artery sling and aberrant subclavian arteries, was not available at the time of these studies. In two patients with a right aortic arch and aberrant left subclavian artery, the arch and its branches were identified and the presence of a left ductal ligament was correctly inferred by correlating the barium swallow appearance. Consequently, in all four cases, adequate information was obtained from the echocardiographic study to enable an anatomical diagnosis, which was confirmed at surgery in each case. In two ventilated infants with pulmonary artery slings (see above) echocardiography was inconclusive. One had severe hyperinflation of both lungs and adequate images could not be obtained. The other had absence of the right lung with gross mediastinal shift. Two patients, both with double aortic arch, underwent echocardiography as the sole preoperative investigation (fig 3, lower). There was no false positive echocardiographic diagnosis of a vascular ring.

Echocardiography was known to have been performed at the referring hospital in six patients. The correct diagnosis of pulmonary artery sling was considered in two patients in whom the left pulmonary artery could not be imaged. In the others, no abnormality of arterial anatomy was identified. In one patient with a double arch the referral for surgery was delayed for one month as a result of the apparently normal echocardiogram.

\section{Angiography}

No vascular compression

Arterial anomaly defined

$\square$ Vascular compression

Inconclusive

Figure 2. Identification of non-specific vascular compression of the trachea and specific arterial anomalies in 40 patients operated 1981-91 from $(A)$ barium swallow examination at the referring hospitals, $(B)$ barium swallow examination at the Hospital for Sick Children, the referring hospitals, $(B)$ other investigation at the referring hospitals, and $(D)$ echocardiography at the Hospital for Sick Children.
Angiography was performed in eight patients. In three it was performed at the referring hospital. One had an elective catheterisation because of ventricular septal defect. A double aortic arch was not identified on the aortogram in the anteroposterior projection. The second patient was catheterised because a vascular ring was suspected, but a double aortic arch was not identified on the aortogram taken in the anteroposterior projection. In the third patient a right 

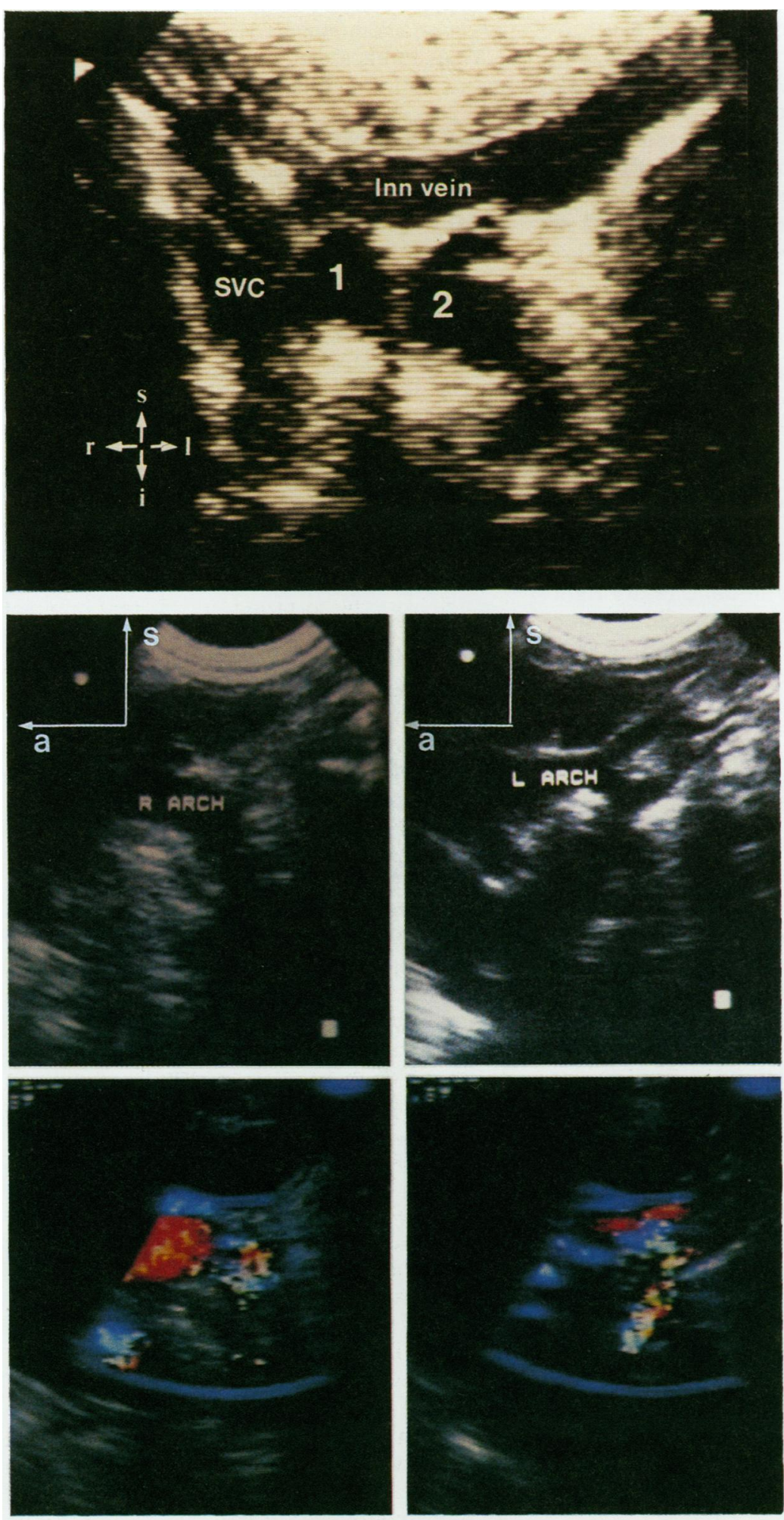

Figure 3 Cross sectional echocardiographic views of double aortic arch. Upper: suprasternal paracoronal view demonstrating two lumens $(1,2)$ of the transverse aortic arch;

Inn=innominate, SVC= superior vena cava. Lower four figures: suprasternal parasagittal views in a different patient demonstrate the lumen of the right arch (upper left) is larger than that of the left arch (upper right), which is confirmed by the respective colour flow Doppler images (lower left and lower right).

aortic arch and anomalous left subclavian artery was correctly identified. A further five patients underwent angiography at the Hospital for Sick Children. Concern over the size and contour of a hypoplastic right pulmonary artery identified on echocardiography was the indication for angiography in one patient with a pulmonary artery sling. The echocardiogram could not exclude a pulmonary artery sling in another infant with absence of the right lung (see above, fig 4). In three others it was considered that the barium studies had not adequately defined the lesion. In one of these a double aortic arch was mistakenly diagnosed at angiography as a right arch with anomalous left subclavian artery because the hypoplastic left sided arch was not seen. A right aortic arch with aberrant left subclavian artery and left duct was correctly diagnosed in another and a pulmonary artery sling diagnosed in the third. These three cases presented in the era before routine echocardiographic assessment and echocardiography had not been performed.

\section{Bronchoscopy}

Bronchoscopy was performed in 11 patients. In seven this was at the referring hospital and

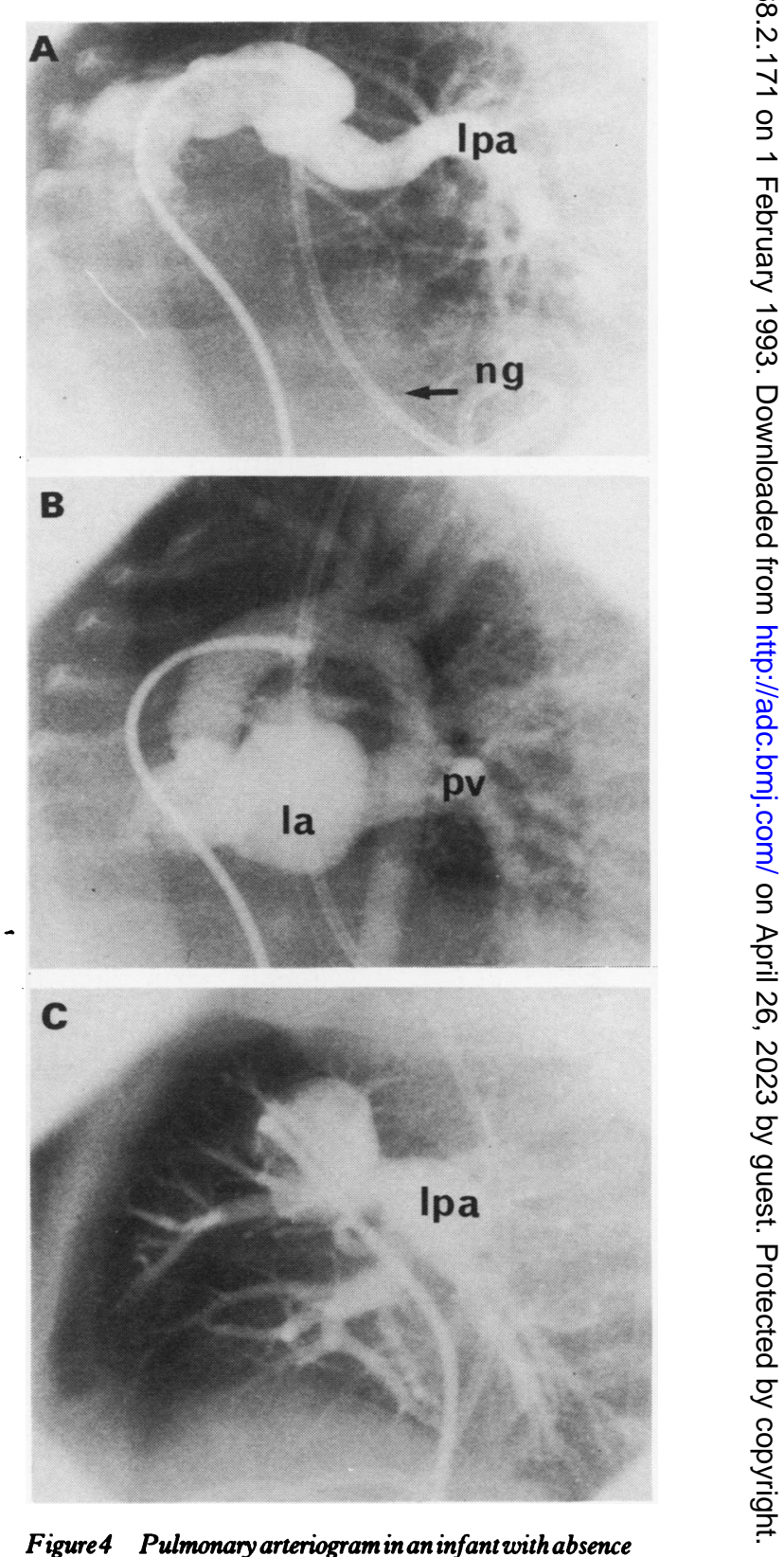

of the right lung and left pulmonary artery passing between the left main bronchus and the oesophagus. Early $(A)$ and late $(B)$ anteroposterior frames. Early lateral frame $(C)$; la $=$ left atrium, lpa $=$ left pulmonary artery, $p v=$ pulmonary vein, $n g=$ nasogastric tube. 
misleading or incomplete information was obtained. An incorrect diagnosis of 'tracheomalacia' or 'no abnormality' was made in $4 / 7$. In the other three patients a severe tracheal stenosis was detected but the extrinsic vascular compression was not recognised. The remaining four patients underwent bronchoscopy at the Hospital for Sick Children, before cardiothoracic referral. Severe tracheal stenosis was noted in all, and extrinsic pulsatile compression recognised in two.

\section{Other investigations}

Computed tomography was performed in three patients at referring hospitals. Pulmonary artery slings were correctly diagnosed in two patients; in the other a double aortic arch was not identified. Orthodox tomography at a referring hospital in a further patient with a right arch, aberrant left subclavian artery, and a left duct mistakenly diagnosed a duplication cyst as the cause of stridor and led to an unnecessary right thoracotomy at the referring hospital. At the Hospital for Sick Children, magnetic resonance imaging correctly identified a pulmonary artery sling in one patient after an inconclusive computed tomogram. Computed tomography was also inconclusive in two other ventilated infants with pulmonary artery sling (see above).

\section{VASCULAR ANOMALIES 1952-91}

The incidence of the differing types of vascular anomaly for the entire group and for the subgroups seen 1952-80 and 1981-91 is shown in the table. In patients seen before 1980 , it was not always possible from the records available to differentiate those with a right aortic arch and retro-oesophageal duct or ligament from those with a right aortic arch, aberrant left subclavian artery, and left duct or liagment. These data include those previously published from our unit. $^{7-11}$ There were seven deaths $(6 \%)$. The mortality decreased, however, from $4 / 29$ (14\%) in the original series $(1952-68),{ }^{7}$ to $1 / 40(3 \%)$ in patients seen 1981-91. The recent death was related to severe tracheal stenosis necessitating tracheoplasty. There was also a late death in a child with tetralogy of Fallot after division of a double aortic arch and a modified Blalock Taussig shunt in the neonatal period, who died suddenly and unexpectedly at home aged 15 months. Associated cardiac anomalies occurred in 11 of $122(10 \%)$ patients: ventricular septal defect $(n=6)$, tetralogy of Fallot $(n=3)$,

Anatomy of vascular anomalies. Figures are number (\%)

\begin{tabular}{llll}
\hline & $\begin{array}{l}1952-91 \\
(n=122)\end{array}$ & $\begin{array}{l}1952-80 \\
(n=82)\end{array}$ & $\begin{array}{c}1981-91 \\
(n=40)\end{array}$ \\
\hline $\begin{array}{l}\text { Double arch } \\
\begin{array}{l}\text { Pulmonary artery sling } \\
\text { Right arch, left retro-oesophageal duct } \\
\text { or ligament }\end{array}\end{array}$ & $\begin{array}{l}73(60) \\
18(15)\end{array}$ & $\begin{array}{l}46(56) \\
11(13)\end{array}$ & $\begin{array}{c}27(67 \cdot 5) \\
7(17 \cdot 5)\end{array}$ \\
$\begin{array}{l}\text { Right arch, aberrant right subclavian } \\
\text { artery, left duct or ligament } \\
\begin{array}{l}\text { Left arch, aberrant right subclavian } \\
\text { artery }\end{array}\end{array}$ & $17(14)$ & $12(15)$ & $5(12 \cdot 5)$ \\
$\begin{array}{l}\text { Innominate artery compression of } \\
\text { trachea* } \\
\text { Left arch, right descending thoracic } \\
\text { aorta, and right duct }\end{array}$ & $9(7)$ & $8(10)$ & $1(2 \cdot 5)$ \\
\hline
\end{tabular}

" [ ] =Additional patients in whom aortopexy was performed by general surgeons because of innominate artery compression of trachea. pulmonary atresia with ventricular septal defect $(n=1)$, and common arterial trunk $(n=1)$.

A left thoracotomy approach was used in all but six patients. A right thoracotomy approach was considered essential in only two (2\%) patients. One of these had a left aortic arch, right descending thoracic aorta and right arterial duct (fig 5). The other had pulmonary atresia with ventricular septal defect, left aortic arch, and an aberrant right subclavian artery from which a right arterial duct originated. A left modified Blalock Taussig shunt was performed in the neonatal period and the right ductal ligament was divided via a right thoracotomy at the age of 14 months. ${ }^{7}$ Four patients underwent median sternotomy: in one patient to elevate the aorta because of tracheal compression by the innominate artery and in three patients with pulmonary artery slings to enable repair using cardiopulmonary bypass (two required extensive tracheal reconstruction in addition).
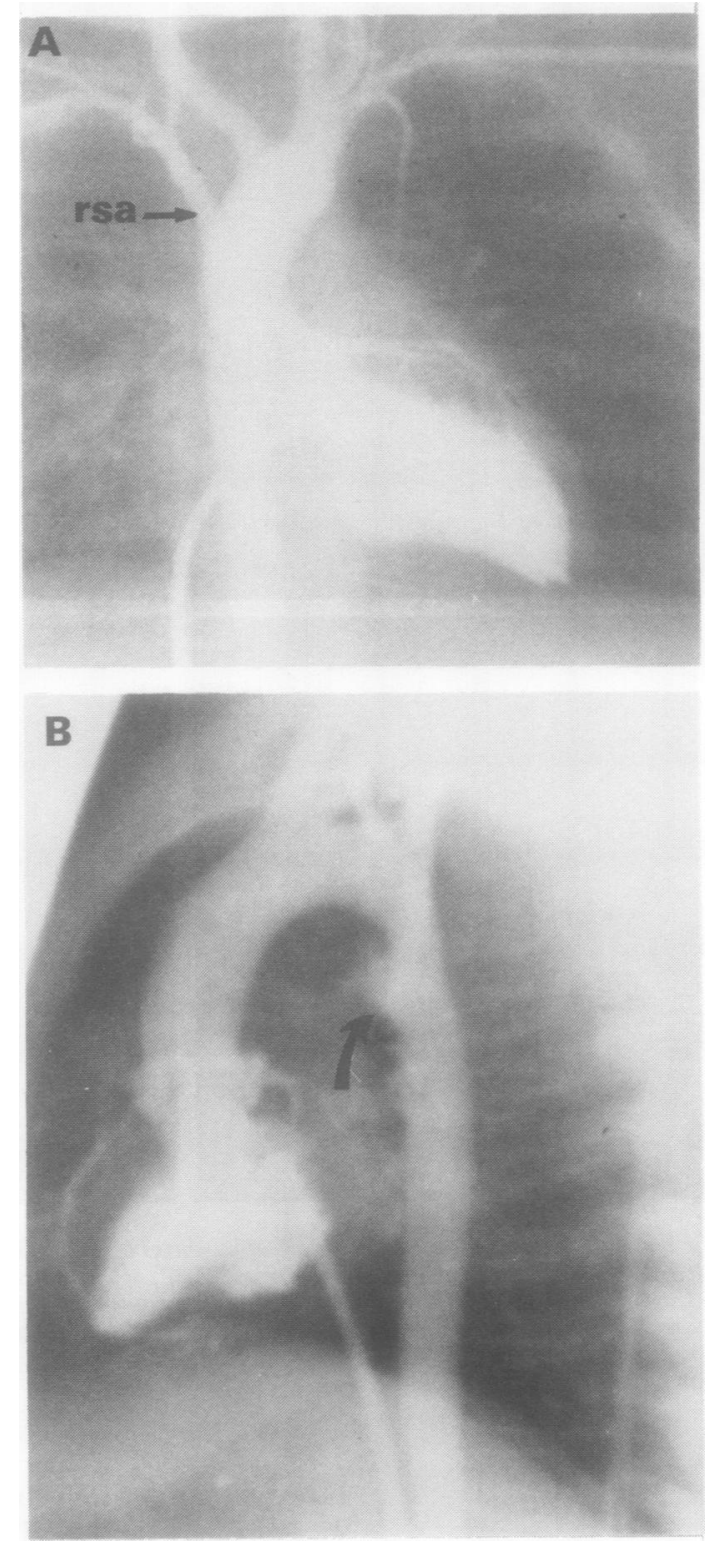

Figure 5 Left ventriculogram in $(A)$ anteroposterior projection and $(B)$ lateral projection in an infant with stridor. The left arch, right descending thoracic aorta, aberrant right subclavian artery (rsa) and right ductal ligament (arrow).

The latter completed the ring and necessitated a right thoracotomy for division. 


\section{Discussion}

ANATOMY OF VASCULAR ANOMALIES

The anatomical spectrum is consistent with other large series, ${ }^{12-15}$ with double aortic arch being easily the most common anomaly.

A left aortic arch with an aberrant right subclavian artery passing posterior to the oesophagus does not result in a vascular 'ring' unless the duct originates from the aberrant subclavian artery, reported in one case, ${ }^{11}$ and is usually an asymptomatic abnormality. Indeed, an iatrogenic 'ring' has been occasionally completed by construction of a palliative prosthetic shunt between the aberrant subclavian artery and the ipsilateral pulmonary artery, without provoking symptoms of tracheal or oesophageal compression. It is likely that the importance of an aberrant subclavian artery in causing vascular compression has been over emphasised in the past, and recognition of this is reflected by the fact that only one patient has undergone surgery for this anomaly since 1980 .

Similarly, an innominate artery causing anterior tracheal compression is not a vascular ring. Four patients with the diagnosis of tracheal compression from an 'anomalous' innominate artery underwent aortopexy before 1980 . However, the term 'anomalous' origin of the innominate artery is incorrect as origin of the innominate artery to the left of the trachea is the rule in normal subjects, ${ }^{16-18}$ and obstructive symptoms seem to be related to an area of localised primary tracheomalacia made evident at bronchoscopy by compression from an anatomically normal innominate artery and arch. $^{16}$ 19 Thus barium swallow, echocardiography, and angiography may be normal, but bronchoscopy will reveal an oblique anterior pulsatile compression of the trachea extending from the left inferiorly to the right superiorly $1-2 \mathrm{~cm}$ above the carina, and it may be possible to obliterate the right sided temporal and radial pulses with anterior bronchoscopic pressure. The change in nomenclature occasioned by better appreciation of pathogenesis has resulted in a change in the pattern of referral. Aortopexy was performed by general surgeons for this condition in 18 patients between 1980 and 1988 at the Hospital for Sick Children (C M Bailey, personal communication). ${ }^{20}$

\section{PREOPERATIVE ASSESSMENT}

Over the last decade barium swallow studies at referring hospitals suggested vascular compression of the oesophagus in all but one patient in whom they were undertaken, although the correct arterial anomaly was identified in only a minority. There were no false positive diagnoses of vascular compression from barium swallow in patients referred to our unit. We do not know if false negative barium swallow diagnoses occurred in addition to the one known case. Barium studies at the Hospital for Sick Children were accurate in the detection of vascular compression except in the only two studies undertaken in ventilated infants. In contrast, echocardiography, bronchoscopy, angiography, and computed tomography at the referring hospitals were generally unhelpful in the identification of vascular anomalies.
It is important to detect preoperatively the rare cases in which there is a left aortic arch with a right sided thoracic descending aorta and a right sided arterial duct or ligament, because a right thoracotomy is then required to divide the ring. ${ }^{21-23}$ The only patient with this type of anatomy in our series of 122 patients (fig 5) was seen before the availability of cross sectional echocardiography. The diagnosis should be apparent from echocardiography as the side of the aortic arch and of the upper descending aorta can be determined. ${ }^{24}$ In our patients, echocardiography after referral correctly indicated the anatomy preoperatively in $20 / 23(87 \%)$, although in four this required correlation with the barium swallow appearance. It is important, however, to contrast the accuracy of echocardiography after referral to echocardiography performed at the referring hospitals where it was unhelpful in the diagnosis of vascular ring on all but two of the six occasions it was performed. In one case referral was inappropriately delayed after a study was presumed to be normal.

Angiography after referral was performed in only five patients over the last decade, which reflects our reliance on barium studies, combined with echocardiography in recent years. None of our patients had a cervical aortic arch, nor an atretic portion to a double arch, which may be difficult to define on echocardiography. ${ }^{+}$In most cases when there is an atretic segment to a double arch, almost always the left arch, ${ }^{2+}$ combining the information from barium swallow and echocardiography with colour flow Doppler should enable prediction of the anatomy in the same manner as with our cases who had a right aortic arch, aberrant left subclavian artery, and left ductal ligament. Ligamentous structures are not demonstrated by echocardiography, angiography, computed tomography, or magnetic resonance imaging. Our experience in one case demonstrates that angiography may fail to differentiate a double aortic arch with hypoplasia or atresia of the left arch between the left carotid artery and left subclavian artery from a right aortic arch with an aberrant left subclavian artery and left duct or ligament. Similarly, angiography may not be able to differentiate a double aortic arch with atresia of the left aortic arch distal to the left subclavian artery from a right aortic arch and left retro oesophageal duct or ligament. ${ }^{2+}$ Fortunately, the surgical approach via a left thoracotomy is the same in each case and the precise anatomy can be identified intraoperatively. ${ }^{25}$ Angiography may be useful in patients with a pulmonary artery sling if there is concern about peripheral pulmonary artery anatomy. A pulmonary artery sling may be referred for surgery on the basis of barium and echocardiographic examination. However, it is important to be aware of the possibility that arterial branches to the right lung may arise from the proximal left pulmonary artery, as seen in a case referred to our unit for surgical opinion, but fully investigated elsewhere and not included in the present series (fig 6). Clearly, the left pulmonary artery must be transected distal to the origin of any branches to the right lung when it is to be reimplanted onto the pulmonary trunk. 


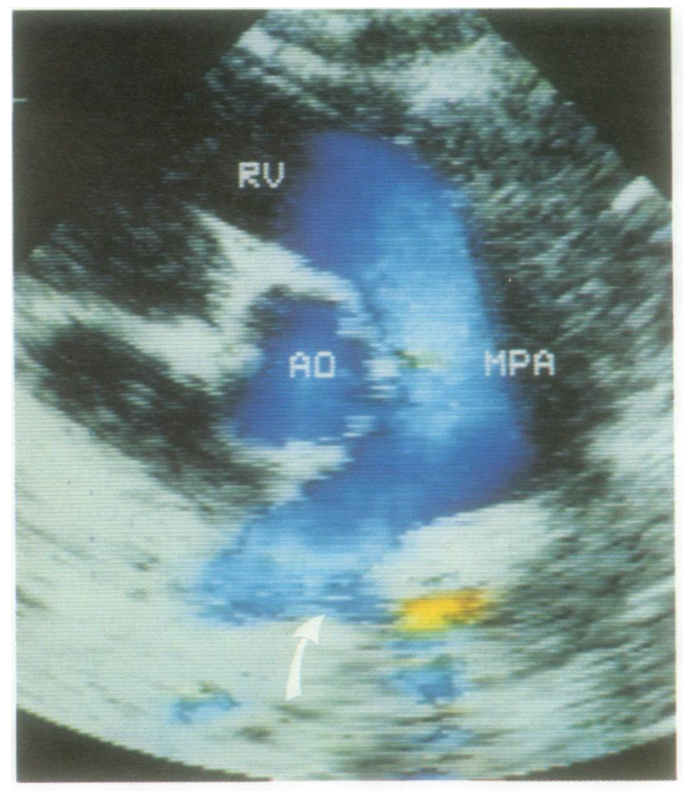

Figure 6 Upper: parasternal echocardiographic view obtained in the short axis of the heart, the ultrasound beam is roughly parallel to a line joining the left shoulder to the right flank and slight caudocranial tilt is applied. Colourflow Doppler demonstratesthe origin of left pulmonary artery (curved arrow) from right pulmonary artery in a patient with pulmonary artery sling. $A O=$ aorta, $M P A=$ main pulmonary artery, $R V=$ right ventricle. Lower: angiography in the same patient demonstrates origin of the right upper lobe pulmonaryartery (triangle) from the proximal left pulmonary artery; $L P A=$ left pulmonary artery, $R P A=$ right pulmonary artery.

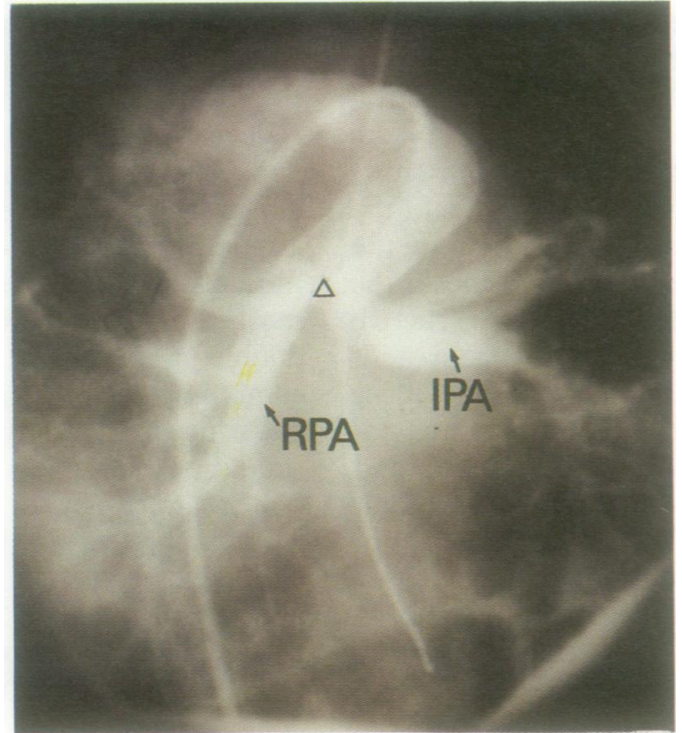

CLINICAL IMPLICATIONS

Barium swallow, to include oblique and lateral views, is the investigation of choice when vascular compression of the trachea is suspected. If there is no evidence of extrinsic compression of the oesophagus on the barium study in an infant or young child with stridor, bronchoscopy might be considered in order to diagnose intrinsic airway abnormality, which may include anterior compression of the trachea by the innominate artery. In the latter situation, aortopexy may be appropriate management. Angiography is unhelpful in the preoperative assessment of these patients. ${ }^{16}$

If the barium study suggests vascular compression of the oesophagus, referral to the surgical centre should not be delayed by further investigation. After referral, echocardiography, ideally with Doppler colour flow imaging, is the most helpful preoperative investigation provided attention is directed to the detailed great artery anatomy in a fully cooperative or sedated subject and adequate ultrasound images are available. A repeat barium study offers little advantage as the precise arterial anomaly can be correctly defined in less than $50 \%$ of examina- tins. Angiography, bronchoscopy, computed tomography, or magnetic resonance imaging can be reserved for specific cases when additional information is required. Further investigations are most likely to be necessary in patients with pulmonary artery slings as the more severe tracheal stenosis in these cases may necessitate intubation, ventilation, and consequent hyperinflation before investigation which renders both barium swallow and echocardiography technically difficult. Bronchoscopy may be helpful in defining the extent of tracheal stenosis and the likelihood of the need for reconstructive tracheal surgery in these severe cases.

We are grateful to Marc de Leval, Duncan Matthew, Donald Shaw, and Christopher Wren for helpful suggestions and Jud Sheat for preparing the manuscript.

Original photograph (fig 6) published courtesy of $\mathrm{Dr} C \mathrm{~W}_{\mathrm{ren}}$.

1 Hommel, cited by Turner W. On irregularities of the pulmonary artery, arch of the aorta and the primary branches of the arch with an attempt to illustrate the mode of origin by a reference of development. Br Foreign Med Chir Rev 1962;30:173-89.

2 Gross RE. Surgical relief for tracheal obstruction from a vascular ring. N Engl Y Med 1945;233:586-90.

Enderlein MA, Silverman NH, Stanger P, Heymann MA. Usefulness of suprasternal notch echocardiography for diagnosis of double aortic arch. Am $\mathcal{Y}$ Cardiol 1986;57: 359-61.

4 Murdison KA, Andrews BAA, Chin AJ. Ultrasonographic display of complex vascular rings. 7 Am Coll Cardiol 1990; 15:1645-53.

5 Kan MN, Nanda NC, Stopa AR. Diagnosis of double aortic arch by cross-sectional echocardiography with Doppler colour flow mapping. Br Heart f 1987;58:284-6.

6 Gnanapragasam JP, Houston AB, Jamieson MPG. Pulmonary artery sling: definitive diagnosis by colour Pulmonary artery sling: definitive diagnosis by colour Doppler flow mapping

7 Lincoln JCR, Deverall PB, Stark J, Aberdeen E, Waterston DJ. Vascular anomalies compressing the oesophagus and trachea. Thorax 1969;24:294-306.

8 Dunn JM, Gordon I, Chrispin AR, de Leval M, Stark J. Early and late results of surgical correction of pulmonary artery sling. Ann Thorac Surg 1979;28:230-8.

9 Roesler M, de Leval M, Chrispin A, Stark J. Surgical management of vascular ring. Ann Surg 1983;197:139-46.

10 Westaby S, Dinwiddie R, Chrispin A, Stark J. Pulmonary artery sling in identical twins. Thorac Cardiovasc Surg 1984; 32:182-3.

11 McKay R, Stark J, de Leval M. Unusual vascular ring in infant with pulmonary atresia and ventricular septal defect. Br Heart F 1982;48:180-3

12 Wychulis AR, Kincaid DW, Weidman WH, Danielson GK. Congenital vascular ring: surgical consideration and results of operation. Mayo Clin Proc 1971;46:182-8

13 Arciniegas E, Hakimi M, Hertzler JH, Farooki ZQ, Green EW. Surgical management of congenital vascular rings. 7 Thorac Cardiovasc Surg 1979;77:721-7.

14 Binet JP , anglois J. Aortic arch anomalies on children and infants. $\mathcal{Y}$ Thorac Cardiovasc Surg 1977;73:248-52.

15 Bertolini A, Pelizza A, Panizzon G, et al. Vascular rings and slings. Foumal of Cardiothoracic Surgery 1987;28:301-12.

16 Cohen D. Tracheopexy aorta tracheal suspension for severe tracheomalacia. Australian Paediatric fournal 1981;17: tracheoma

17 Moes CAF, Izukawa T, Trusler GA. Innominate artery compression of the trachea. Archives of Otolaryngology 1975 101:733-8.

18 Mustard WT, Bayliss CE, Fearon B, Pelton D, Trusler GA Tracheal compression by the innominate artery in children. Ann Thorac Surg 1969;8:312-9.

19 Cohen D, Greenholz SK, Karrer FM, Lilly JR. Contemporary surgery of tracheomalacia. F Pediatr Surg 1986; 21:511-4.

20 Malone PS, Kiely EM. Role of aortopexy in the management of primary tracheomalacia and tracheobronchomalacia. of primary tracheomalacia a

21 Berman W, Yabek SM, Dillon TM, Neal JF, Burstein J. Vascular ring due to left aortic arch and right descending Vascular ring due to left aortic arch

22 McFaul RD, Millard P, Nowicki E. Vascular rings necessitating right thoracotomy. $\mathcal{F}$ Thorac Cardiovasc Surg 1981;82 306-9.

23 Whitman G, Stephenson LW, Weingberg P. Vascular ring left cervical aortic arch, right descending aorta and righ liagmentum arteriosum. I Thorac Cardiovasc Surg 1982;83: $311-5$.

24 Freedom RM, Culham JAG, Moes CAF. Vascular rings and associated anomalies. Angiography of congenital hear disease. New York: Macmillan, 1984:487-511.

25 de Leval M. Vascular rings. In: Stark J, de Leval M, eds. Surgery for congenital heart defects. London: Grune and Stratton, 1983:227-34. 